\title{
Effect of STARD3 gene polymorphism on carcass traits and fatty acid composition in Japanese Black cattle
}

\author{
Hiroto Kigoshi $^{1}$, Fuki Kawaguchi ${ }^{1}$, Kenji Oyama ${ }^{2}$, Hideyuki Mannen ${ }^{1}$, Shinji Sasazaki ${ }^{1}$ \\ ${ }^{1}$ Laboratory of Animal Breeding and Genetics, Graduate School of Agricultural \\ Science, Kobe University, Kobe 6578501, Japan \\ ${ }^{2}$ Food Resources Education \& Research Center, Kobe University, Kasai 6752103, Japan
}

\begin{abstract}
In this study, we focused on the STARD3 gene which is involved in cholesterol distribution and homeostasis in cells, and investigated the effects of the STARD3 gene polymorphism on carcass traits and fatty acid composition. We searched for polymorphisms in the STARD3 gene by whole-genome resequencing and selected a missense S396L from 280 detected polymorphisms. We genotyped S396L polymorphism for Japanese Black cattle population $(n=441)$ and then analyzed the effects on carcass traits and fatty acid composition. As a result, the polymorphism showed strong association with fatty acid composition (C14:0, C16:0, C18:1, SFA and MUFA) ( $\mathrm{p}<0.0001)$. Although further research is warranted to clarify whether S396L directly impacts fatty acid composition, it is obvious that there is a novel QTL for fatty acid composition on BTA19 in the Japanese Black cattle. In conclusion, these results contribute to the identification of a novel causative mutation on BTA19 for fatty acid composition and development of a useful DNA marker for improving the beef quality of Japanese Black cattle.
\end{abstract}

Key words: beef; STARD3; fatty acid composition

\section{INTRODUCTION}

STARD3 is a transmembrane protein that is localized on late endosomes (Clark 2012) and plays a role in the maintenance of cholesterol distribution and homeostasis in cells by transporting cholesterol from the endoplasmic reticulum (ER) to endosomes via its cholesterol binding domain (Aply et al. 2001; Wilhelm et al. 2017). Excess cholesterol that remains in the ER is esterified to lipid droplets for storage (Roger et al. 2015). Therefore, STARD3 has been suggested to be one of the factors involved in regulating intercellular lipid accumulation.

Moreover, STARD3 affects the expression level of some genes involved in energy metabolism. Soffientini et al. (2014) observed different expression levels of CYP2E1, $G 6 P C$, and PAI1 genes in STARD3-overexpressing cells vs. control cells, suggesting the association of STARD3 with diseases such as glycaemia, fatty liver, and obesity. Zhou et al. (2018) reported that STARD3 may have an important role in the differentiation of adipocytes and adipogenesis in preadipocytes as it affects the expression levels of PPAR $\gamma$ and $\mathrm{C} / \mathrm{EBP} \alpha$ genes in preadipocytes. These effects on the expression of genes seem to be due to changes in STARD3- mediated cholesterol metabolism, suggesting that STARD3 gene affects fat metabolism.

Beef cattle have been genetically modified for improved beef quantity and quality traits. Beef breed traits such as carcass weight (CW), rib eye area (REA), and subcutaneous fat thickness (SFT) are commonly modified for improving beef quality (Boykin et al. 2017; Riley et al. 2002; Wheeler et al. 1996). In addition, beef marbling and fatty acid composition have also been targeted as traits for determining beef quality (Harris et al. 2018; Mannen et al. 2011). DNA markers have been developed corresponding to each targeted trait for genetic improvement. In several cases, these DNA markers demonstrated significant association with beef traits, probably because many traits are influenced by fat metabolism. For instance, an amino acid substitution in the leptin gene, which controls energy homeostasis through food intake repression, is significantly associated with several beef traits such as carcass weight, back fat

Correspondence: Shinji Sasazaki, Laboratory of Animal Breeding and Genetics, Graduate School of Agricultural Science, Kobe University, Nada, Kobe, 657-8501, Japan. (e-mail: sasazaki@kobe-u.ac.jp) 
thickness, rib eye area, and fatty acid composition (Corva et a1. 2009; Kawaguchi et al. 2017; Tian et al. 2013). Therefore, we aimed to identify polymorphisms significantly associated with beef traits that are related to fat metabolism such as polymorphisms occurring in STARD3. Accordingly, we investigated the association between STARD3 gene polymorphisms and various beef traits in Japanese Black cattle.

\section{MATERIALS AND METHODS}

\section{Animals}

For whole-genome resequencing, eight animals were selected from a Japanese Black cattle population comprising 1836 animals bred in Hyogo Prefecture. These eight animals were the progenies of different eight sires. Genomic DNA was extracted from 50-mg samples of longissimus cervicis muscle of each animal using the standard phenolchloroform method.

For genotyping, Japanese Black cattle population [average age ( \pm standard deviation), $31.83 \pm 1.37$ months] of 441 animals from Hyogo Prefecture was used. Carcass traits such as CW, REA, rib thickness (RT), SFT, yield estimate, and BMS were measured by official graders of the Japan Meat Grading Association (Table 1). Genomic DNA was extracted in the aforementioned manner. Intramuscular fat samples were collected from the longissimus thoracis muscle.

The muscle and fat samples from each animal were collected under routine work with breeder' $\mathrm{s}$ approval. No experiment was performed on the animals in the current study.

\section{Whole-genome resequencing}

DNA degradation and concentration were monitored using spectrometry, fluorometry, and $1 \%$ agarose gel electrophoresis. A paired-end library, with a read length of $150 \mathrm{bp}$, was constructed using high-quality DNA for each animal. Whole-genome sequencing was performed using the HiSeq X Five Sequencing System (Illumina Inc., San Diego, CA, USA). Sequencing data were normalized using the Genedata Expressionist 9.1.4a. These reads were mapped to the reference cattle genome sequence (UCSC bosTau8) downloaded from the UCSC Genome Browser assembly (https://genome-asia.ucsc.edu/cgi-bin/hgGateway) using BWA-MEM 0.7.12. and PCR duplicates were excluded
Table 1. Summary statistics of carcass traits and fatty acid composition in a Japanese Black cattle population $(n=441)$

\begin{tabular}{|c|c|c|c|c|c|}
\hline & \multicolumn{5}{|c|}{$n=441$} \\
\hline & Mean & SD & Min. & Max. & heritability \\
\hline Slaughter age & 31.69 & 1.24 & 28.44 & 35.87 & - \\
\hline Slaughter year & \multicolumn{4}{|c|}{$2010-2011$} & - \\
\hline $\begin{array}{l}\text { Proportion of sex } \\
\text { (steer / cow) }\end{array}$ & \multicolumn{4}{|c|}{$79.8 / 20.2$} & - \\
\hline Number of sires & \multicolumn{4}{|c|}{7} & - \\
\hline \multicolumn{6}{|l|}{ Trait } \\
\hline Carcass weight (kg) & 393.2 & 38.6 & 301 & 472.4 & 0.451 \\
\hline Rib eye area $\left(\mathrm{cm}^{2}\right)$ & 53.54 & 6.36 & 32.92 & 76.5 & 0.421 \\
\hline Rib thickness (cm) & 6.55 & 0.65 & 4.8 & 8.5 & 0.323 \\
\hline $\begin{array}{l}\text { Subcutaneous } \\
\text { fat thickness (cm) }\end{array}$ & 2.3 & 0.66 & 1 & 4.7 & 0.428 \\
\hline Yield estimate (\%) & 73.5 & 1.1 & 69.6 & 77.9 & 0.593 \\
\hline BMS & 5.66 & 1.76 & 2 & 11 & 0.388 \\
\hline \multicolumn{6}{|l|}{$\begin{array}{l}\text { Fatty acid } \\
\text { composition }\end{array}$} \\
\hline C14:0 & 1.96 & 0.34 & 1.08 & 3.13 & 0.726 \\
\hline C14:1 & 0.94 & 0.23 & 0.33 & 1.7 & 0.64 \\
\hline C16:0 & 21.9 & 2.23 & 15.86 & 29.08 & 0.496 \\
\hline C16:1 & 4.36 & 0.72 & 2.63 & 6.72 & 0.528 \\
\hline C18:0 & 10.87 & 1.81 & 4.56 & 19.95 & 0.548 \\
\hline C18:1 & 54.76 & 3.01 & 45.45 & 64.01 & 0.423 \\
\hline C18:2 & 2.17 & 0.43 & 1.2 & 3.69 & 0.342 \\
\hline MUFA & 61.43 & 3.53 & 49.7 & 71.64 & 0.427 \\
\hline SFA & 36.3 & 3.54 & 25.68 & 48.88 & 0.429 \\
\hline
\end{tabular}

using the Picard 2.2.4. Moreover, GATK 3.6 (2016-12-08-g1c2527f) was used to detect polymorphisms by comparing genome sequences, including the reference genome sequence. The polymorphisms were annotated to the reference genome sequence (NCBI RefSeq) based on their location (intron, exon, untranslated region, upstream, downstream, splice site, and intergenic region) and characteristics (synonymous/ non-synonymous amino acid replacement, gain/loss of start/stop site, and frameshift mutations) using the SnpEff v4.2.

\section{Candidate polymorphism extraction}

Focusing on the STARD3 gene (chr19: 4065924040693948), the missense polymorphism, S396L (rs134877666), was selected as the candidate polymorphism from all polymorphisms detected using whole-genome resequencing within the STARD3 gene.

\section{Genotyping using PCR-RFLP}

S396L was genotyped using PCR-RFLP, in which the primer sets for PCR amplification were designed based on the GenBank sequence (AC_000177.1) using OLIGO 7.41 (F: 5'- AGG AGG ATT TGA GCA CCC CAT -3', R: 5'- CAA GGT CAC ACA GCA CAC TCC -3'). PCR products for 
S396L were digested using Msc1 (5'- TGG/CCA -3') and subsequently separated using electrophoresis in $2 \%$ agarose gel, stained with ethidium bromide and visualized under UV light.

\section{Statistical analysis}

The effect of the STARD3 polymorphism S396L on carcass traits and fatty acid composition was statistically analyzed using analysis of variance (ANOVA) and Tukey's honestly significant difference test. The analytical model included the effect of shipment year; shipment month; and linear and quadratic covariates for age at slaughter, sire, sex, and genotype without interactions.

\section{RESULTS}

The STARD3 genomic sequence is 24,694 bp long and consists of 15 exons with 14 introns. The length of coding sequence is $1,347 \mathrm{bp}$ coding 448 amino acids. A total of 280 polymorphisms were detected within the STARD3 gene using whole-genome resequencing (Table S1). Of these, most (204) were detected in intron regions, 25 SNPs upstream, 37 SNPs downstream, 2 SNPs in 5'UTR, 5 SNPs in 3 'UTR, and $1 \mathrm{SNP}$ in the splice region and 5 SNPs were synonymous and $1 \mathrm{SNP}$ was a missense polymorphism (S396L).

Genotyping of the missense polymorphism in Japanese Black cattle population $(n=441)$ revealed that the $C$ and $T$ allele frequencies were 0.553 and 0.447 , respectively (Table 2). Statistical analysis revealed significant associations between S396L and RT, C14:0, C16:0, C18:1, MUFA, and SFA $(\mathrm{p}<0.05)$. The $\mathrm{S} 396 \mathrm{~L}$ polymorphism was particularly strongly associated with fatty acid composition $(\mathrm{p}<0.0001)$. The CC genotype had a significantly lower percentage than the TT genotype in C14:0, C16:0, and SFA $(0.15,1.39$, and 2.20 , respectively) and had a significantly higher percentage than the TT genotype in C18:1 and MUFA (1.85 and 2.19, respectively).

\section{DISCUSSION}

We focused on the STARD3 gene involved in lipid metabolism and used whole-genome resequencing to identify polymorphisms in the STARD3 gene. Among 280 SNPs detected in the STARD3 gene, some polymorphisms, such as those in $5^{\prime} \mathrm{UTR}$ and $3^{\prime} \mathrm{UTR}$, were considered to be candidate polymorphism to affect gene function. However, in the current study, we selected the missense polymorphism (S396L) as a candidate polymorphism and subsequently investigated the association between this polymorphism, carcass traits and fatty acid composition for Japanese Black cattle population from Hyogo Prefecture. We will need to consider the remaining candidate polymorphisms and conduct similar analysis to investigate the effect of the polymorphisms on traits.

Based on the results of the association analysis, S396L

Table 2. Genotype frequency of STARD3 gene polymorphism S396 L and effect on carcass traits and fatty acid composition

\begin{tabular}{|c|c|c|c|c|c|c|c|c|c|c|}
\hline \multirow[b]{2}{*}{ Carcass weight (kg) } & \multirow{2}{*}{$\begin{array}{c}\begin{array}{c}\text { ANOVA } \\
\text { P value }\end{array} \\
\mathbf{0 . 1 0 3 6}\end{array}$} & \multicolumn{3}{|c|}{$\begin{array}{c}\mathrm{CC} \\
\mathrm{n}=141 \\
(\mathbf{0 . 3 2 0})\end{array}$} & \multicolumn{3}{|c|}{$\begin{array}{c}\mathbf{C T} \\
\mathbf{n = 2 0 6} \\
(\mathbf{0 . 4 6 7 )}\end{array}$} & \multicolumn{3}{|c|}{$\begin{array}{c}\text { TT } \\
\mathbf{n = 9 4} \\
(\mathbf{0 . 2 1 7})\end{array}$} \\
\hline & & 383.7 & \pm & 3.52 & 375.7 & \pm & 3.14 & 381.4 & \pm & 4.29 \\
\hline Rib eye area $\left(\mathrm{cm}^{2}\right)$ & 0.243 & 51.82 & \pm & 0.68 & 50.75 & \pm & 0.61 & 51.85 & \pm & 0.83 \\
\hline Rib thickness (cm) & 0.0143 & $6.58^{\mathrm{ab}}$ & \pm & 0.065 & $6.45 a$ & \pm & 0.058 & $6.67^{b}$ & \pm & 0.08 \\
\hline Subcutaneous fat thickness $(\mathrm{cm})$ & 0.2701 & 2.47 & \pm & 0.062 & 2.39 & \pm & 0.055 & 2.34 & \pm & 0.075 \\
\hline Yield estimate (\%) & 0.1573 & 73.51 & \pm & 0.11 & 73.47 & \pm & 0.097 & 73.72 & \pm & 0.13 \\
\hline BMS & 0.4135 & 5.52 & \pm & 0.18 & 5.61 & \pm & 0.16 & 5.53 & \pm & 0.22 \\
\hline Fatty acid composition & & & \pm & & & \pm & & & \pm & \\
\hline C14:0 & 0.0088 & $1.89^{\mathrm{a}}$ & \pm & 0.034 & $1.95^{\mathrm{ab}}$ & \pm & 0.03 & $2.04^{b}$ & \pm & 0.041 \\
\hline C14:1 & 0.2705 & 0.98 & \pm & 0.023 & 0.96 & \pm & 0.02 & 0.93 & \pm & 0.027 \\
\hline C16:0 & $<0.0001$ & $21.11^{\mathrm{a}}$ & \pm & 0.21 & $21.66^{\mathrm{a}}$ & \pm & 0.19 & $22.50^{b}$ & \pm & 0.027 \\
\hline C16:1 & 0.1153 & 4.56 & \pm & 0.068 & 4.46 & \pm & 0.061 & 4.36 & \pm & 0.083 \\
\hline C18:0 & 0.0712 & 10.42 & \pm & 0.18 & 10.64 & \pm & 0.16 & 10.99 & \pm & 0.22 \\
\hline C18:1 & $<0.0001$ & $55.83^{\mathrm{a}}$ & \pm & 0.28 & $55.12^{\mathrm{a}}$ & \pm & 0.25 & $53.98^{b}$ & \pm & 0.34 \\
\hline C18:2 & 0.7428 & 2.14 & \pm & 0.044 & 2.17 & \pm & 0.039 & 2.13 & \pm & 0.054 \\
\hline MUFA & $<0.0001$ & $62.81^{\mathrm{a}}$ & \pm & 0.33 & $61.94^{a}$ & \pm & 0.29 & $60.62^{b}$ & \pm & 0.4 \\
\hline SFA & $<0.0001$ & $34.95^{\mathrm{a}}$ & \pm & 0.33 & $35.79^{a}$ & \pm & 0.29 & $37.15^{b}$ & \pm & 0.4 \\
\hline
\end{tabular}

Means with different superscript $(a, b)$ are significantly different between genotypes. 
was found to be significantly associated with RT and fatty acid composition (C14:0, C16:0, C18:1, MUFA, and SFA). Although a significant association between S396L and RT was observed, no significant difference between the $\mathrm{CC}$ and TT genotypes was noted. Moreover, no additive genetic effect among genotypes was observed. This weak association suggested that the STARD3 gene does not have a direct effect on RT and further investigation is warranted to diagnose the effect of the SNP on RT. However, a stronger effect was observed between S396L and fatty acid composition. The CC genotype had a significantly higher percentage than the TT genotype in C18:1 and MUFA and a lower percentage than the TT genotype in C14:0, C16:0, and SFA. These results suggest that $\mathrm{S} 396 \mathrm{~L}$ affects the function of the STARD3 gene and influences fatty acid composition.

Horie et al. (2013) reported that under cholesterol-rich conditions, acetyl-CoA is preferred as a substrate for fatty acid production; however, under sterol-depleted conditions, acetyl-CoA is preferred as a substrate for cholesterol production in mice. Since the STARD3 gene is involved in the transportation of cholesterol, a mutation in the STARD3 gene would alter the amount of cholesterol in the liver (Ugo et al. 2014). In cattle, fatty acid composition might also be affected by changes in the amount of cholesterol due to a polymorphism in the $S T A R D 3$ gene. However, the relationship between the $S T A R D 3$ gene and fatty acid composition has not yet been investigated; therefore, further research is warranted to clarify whether S396L directly impacts fatty acid composition.

As an alternative hypothesis, S396L could be high in LD with a causative mutation. For instance, the fatty acid synthase (FASN) gene, located near the STARD3 gene (chr19:51Mb), is considered as a causative gene for influencing fatty acid composition in many cattle breeds including Japanese Black cattle (Abe et al. 2008; Bartoň et al. 2016). For this reason, the associations observed in this study were considered to be due to the effects of the FASN gene. However, alleles of the FASN gene are almost fixed (minor allele frequency of 0.09), in the Japanese Black cattle population of Hyogo Prefecture, which was used in this study (Ookura et al. 2013). Therefore, the associations observed in this study would not be due to the FASN gene and it suggests that there would be novel QTL for fatty acid composition on BTA19 (bovine chromosome 19) in the Japanese Black cattle population of Hyogo Prefecture.

\section{CONCLUSIONS}

These results contribute to the identification of a novel causative mutation on BTA19 for fatty acid composition and development of a useful DNA marker for improving the beef quality of Japanese Black cattle.

\section{REFERENCES}

Abe T, Saburi J, Hasebe H, Nakagawa T, Misumi S, Nade T, Nakajima H, Shoji N, Kobayashi M, Kobayashi E. 2009. Novel Mutations of the FASN Gene and Their Effect on Fatty Acid Composition in Japanese Black Beef. Biochemical Genetics, 47:397-411

Alpy F, Stoeckel ME, Dierich A, Escola JM, Wendling C, Chenard MP, Vanier MT, Gruenberg J, Tomasetto C, Rio MC. 2001. The steroidogenic acute regulatory protein homolog MLN64, a late endosomal cholesterolbinding protein. The Journal of Biological Chemistry, 276(6):4261-4269.

Bartoň L, Bureš D, Kott T, Řehák D. 2016. Associations of polymorphisms in bovine DGAT1, FABP4, FASN, and PPARGC1A genes with intramuscular fat content and the fatty acid composition of muscle and subcutaneous fat in Fleckvieh bulls. Meat Science, 114:18-23.

Boykin CA, Eastwood LC, Harris MK, Hale DS, Kerth CR, Griffin DB, Arnold AN, Hasty JD, Belk KE, Woerner DR, Delmore RJ, Martin JN, VanOverbeke DL, Mafi GG, Pfeiffer MM, Lawrence TE, McEvers TJ, Schmidt TB Maddock RJ, Johnson DD, Carr CC, Scheffler JM, Pringle TD, Stelzleni AM, Gottlieb J, Savell JW. 2017. National Beef Quality Audit-2016: In-plant survey of carcass characteristics related to quality, quantity, and value of fed steers and heifers. Journal of Animal Science, 95(7):2993-3002.

Clark BJ. 2012. The mammalian START domain protein family in lipid transport in health and disease. Journal of Endocrinology, 212(3):257-275.

Corva PM, Fernández Macedo GV, Soria LA, Papaleo Mazzucco J, Motter M, Villarreal EL, Schor A, Mezzadra CA, Melucci LM, Miquel MC. 2009. Effect of leptin gene polymorphisms on growth, slaughter and meat quality traits of grazing Brangus steers. Genetics and Molecular Research, 8(1):105-116.

Harris CL, Wang B, Deavila JM, Busboom JR, Maquivar M, Parish SM, McCann B, Nelson ML, Du M. 2018. Vitamin A administration at birth promotes calf growth 
and intramuscular fat development in Angus beef cattle. Journal of Animal Science and Biotechnology, 9:55.

Horie T, Nishino T, Baba O, Kuwabara Y, Nakao T, Nishiga M, Usami S, Izuhara M, Sowa N, Yahagi N, et al. 2013. MicroRNA-33 regulates sterol regulatory elementbinding protein 1 expression in mice. Nature Communications, 4:2883

Kawaguchi F, Okura K, Oyama K, Mannen H, Sasazaki S. 2017. Identification of leptin gene polymorphisms associated with carcass traits and fatty acid composition in Japanese Black cattle. Animal Science Journal, 88(3):433-438.

Mannen H. 2011. Identification and utilization of genes associated with beef qualities. Animal Science Journal, 82(1):1-7.

Ookura K, Akiyama T, Yoshida E, Fukushima M, Iwamoto E, Oka A, Matsumoto Y, Sasazaki S, Oyama K, Mannen H. 2013. Effects of genes on economically important traits of Japanese Black cattle in Hyogo population. Nihon Chikusan Gakkaiho 84, 157-162.

Riley DG, Chase CC Jr, Hammond AC, West RL, Johnson DD, Olson TA, Coleman SW. 2002. Estimated genetic parameters for carcass traits of Brahman cattle. Journal of Animal Science, 80(4):955-962.

Rogers MA, Liu J, Song BL, Li BL, Chang CC, Chang TY. 2015. Acyl-CoA:cholesterol acyltransferases (ACATs/ SOATs): Enzymes with multiple sterols as substrates and as activators. The Journal of Steroid Biochemistry and Molecular Biology, 151:102-107.

Soffientini U, Caridis AM, Dolan S1, Graham A. 2014. Intracellular cholesterol transporters and modulation of hepatic lipid metabolism: Implications for diabetic dyslipidaemia and steatosis. Biochimica et Biophysica Acta, 1842(10):1372-1382.

Tian J, Zhao Z, Zhang L, Zhang Q, Yu Z, Li J, Yang R. 2013. Association of the leptin gene E2-169T $>C$ and E3-299T $>$ A mutation with carcass and meat quality traits of the Chinese Simmental-cross steers. Gene, 518(2):443-448.

Wheeler TL, Cundiff LV, Koch RM, Crouse JD. 1996. Characterization of biological types of cattle (Cycle IV): carcass traits and longissimus palatability. The Journal of Animal Science, 74(5):1023-1035.

Wilhelm LP, Wendling C, Védie B, Kobayashi T, Chenard MP, Tomasetto C, Drin G, Alpy F. 2017. STARD3 mediates endoplasmic reticulum-to-endosome cholesterol transport at membrane contact sites. The EMBO Journal, 36(10):1412-1433.

Zhou X, Gao H, Guo Y, Chen Y, Ruan XZ. 2018. Knocking down Stard3 decreases adipogenesis with decreased mitochondrial ROS in 3T3-L1 cells. Biochemical and Biophysical Research Communications, 504(2):387392. 
KIGOSHI et al.

Table S1 List of all polymorphisms deteced in STARD3 gene

\begin{tabular}{|c|c|c|c|c|c|c|}
\hline POSITION & REF & ALT & Change_Type & Annotation & HGVS.c & HGVS.p \\
\hline 40659318 & A & G & SNP & upstream_gene_variant & c. $-18011 \mathrm{~A}>\mathrm{G}$ & \\
\hline 40659357 & G & A & SNP & upstream_gene_variant & c. $-17972 \mathrm{G}>\mathrm{A}$ & \\
\hline 40659380 & $\mathrm{C}$ & $\mathbf{T}$ & SNP & upstream_gene_variant & c. $-17949 \mathrm{C}>\mathrm{T}$ & \\
\hline 40659430 & $\mathrm{C}$ & $\mathbf{T}$ & SNP & upstream_gene_variant & c. $-17899 \mathrm{C}>\mathrm{T} ; \mathrm{c} .241+1308 \mathrm{C}>\mathrm{T}$ & \\
\hline 40659568 & G & A & SNP & upstream_gene_variant & c. $-17761 \mathrm{G}>\mathrm{A}$ & \\
\hline 40660284 & $\mathbf{T}$ & G & SNP & upstream_gene_variant & c. $-17045 \mathrm{~T}>\mathrm{G}$ & \\
\hline 40660422 & СССТTTTCTCTGAACCCCCTCGT & $\mathrm{C}$ & DEL & upstream_gene_variant & c.-16906_-16885delCCTTTTCTCTGAACCCCCTCGT & \\
\hline 40660681 & G & A & SNP & upstream_gene_variant & c. $-16648 \mathrm{G}>\mathrm{A}$ & \\
\hline 40660855 & C & $\mathbf{T}$ & SNP & upstream_gene_variant & c. $-16474 \mathrm{C}>\mathrm{T}$ & \\
\hline 40660888 & G & A & SNP & upstream_gene_variant & c. $-16441 \mathrm{G}>\mathrm{A}$ & \\
\hline 40660947 & $\mathrm{C}$ & G & SNP & upstream_gene_variant & c. $-16382 \mathrm{C}>\mathrm{G}$ & \\
\hline 40661209 & $\mathrm{C}$ & $\mathbf{T}$ & SNP & upstream_gene_variant & c. $-16120 \mathrm{C}>\mathrm{T}$ & \\
\hline 40661210 & A & G & SNP & upstream_gene_variant & c. $-16119 \mathrm{~A}>\mathrm{G}$ & \\
\hline 40661887 & G & A & SNP & upstream_gene_variant & c. $-15442 \mathrm{G}>\mathrm{A}$ & \\
\hline 40662062 & G & $\mathrm{C}$ & SNP & upstream_gene_variant & c. $-15267 \mathrm{G}>\mathrm{C}$ & \\
\hline 40662188 & A & C & SNP & upstream_gene_variant & c. $-15141 \mathrm{~A}>\mathrm{C}$ & \\
\hline 40662205 & CCATTA & $\mathrm{C}$ & DEL & upstream_gene_variant & c.-15123_-15119delCATTA & \\
\hline 40662357 & G & A & SNP & upstream_gene_variant & c. $-14972 \mathrm{G}>\mathrm{A}$ & \\
\hline 40662478 & $\mathrm{C}$ & $\mathrm{T}$ & SNP & upstream_gene_variant & c. $-14851 C>T$ & \\
\hline 40662735 & $\mathbf{T}$ & $\mathrm{C}$ & SNP & upstream_gene_variant & c. $-14594 \mathrm{~T}>\mathrm{C}$ & \\
\hline 40663062 & A & G & SNP & upstream_gene_variant & c. $-14267 \mathrm{~A}>\mathrm{G}$ & \\
\hline 40663851 & G & A & SNP & upstream_gene_variant & c. $-13478 \mathrm{G}>\mathrm{A}$ & \\
\hline 40663878 & $\mathrm{C}$ & $\mathbf{A}$ & SNP & upstream_gene_variant & c. $-13451 C>A$ & \\
\hline 40664028 & $\mathrm{C}$ & $T$ & SNP & upstream_gene_variant & c. $-13301 \mathrm{C}>\mathrm{T}$ & \\
\hline 40664255 & G & $\mathrm{C}$ & SNP & upstream_gene_variant & c. $-13074 \mathrm{G}>\mathrm{C}$ & \\
\hline 40664307 & $\mathbf{A}$ & G & SNP & 5_prime_UTR_variant & c. $-142 \mathrm{~A}>\mathrm{G}$ & \\
\hline 40664417 & $\mathrm{C}$ & A & SNP & intron_variant & c. $-52+20 C>A$ & \\
\hline 40664446 & G & A & SNP & intron_variant & c. $-52+49 \mathrm{G}>\mathrm{A}$ & \\
\hline 40664461 & $\mathbf{T}$ & $\mathrm{C}$ & SNP & intron_variant & c. $-52+64 \mathrm{~T}>\mathrm{C}$ & \\
\hline 40664642 & $\mathrm{C}$ & $\mathrm{T}$ & SNP & intron_variant & c. $-52+245 \mathrm{C}>\mathrm{T}$ & \\
\hline 40664721 & $\mathbf{A}$ & G & SNP & intron_variant & c. $-52+324 \mathrm{~A}>\mathrm{G}$ & \\
\hline 40664824 & G & A & SNP & intron_variant & c. $-52+427 \mathrm{G}>\mathrm{A}$ & \\
\hline 40665099 & A & G & SNP & intron_variant & c. $-52+702 A>G$ & \\
\hline 40665159 & $\mathrm{C}$ & A & SNP & intron_variant & c. $-52+762 \mathrm{C}>\mathrm{A}$ & \\
\hline 40665315 & A & G & SNP & intron_variant & c. $-52+918 \mathrm{~A}>\mathrm{G}$ & \\
\hline 40665390 & $\mathrm{~T}$ & A & SNP & intron_variant & c. $-52+993 \mathrm{~T}>\mathrm{A}$ & \\
\hline 40665394 & G & A & SNP & intron_variant & c. $-52+997 \mathrm{G}>\mathrm{A}$ & \\
\hline 40665435 & $\mathrm{C}$ & G & SNP & intron_variant & c. $-52+1038 \mathrm{C}>\mathrm{G}$ & \\
\hline 40665538 & G & $T$ & SNP & intron_variant & c. $-52+1141 \mathrm{G}>\mathrm{T}$ & \\
\hline 40665788 & $T$ & A & SNP & intron_variant & c. $-52+1391 \mathrm{~T}>\mathrm{A}$ & \\
\hline 40665838 & $T$ & C & SNP & intron_variant & c. $-52+1441 \mathrm{~T}>\mathrm{C}$ & \\
\hline 40665916 & C & A & SNP & intron_variant & c. $-52+1519 \mathrm{C}>\mathrm{A}$ & \\
\hline 40665951 & G & A & SNP & intron_variant & c. $-52+1554 \mathrm{G}>\mathrm{A}$ & \\
\hline 40665990 & G & A & SNP & intron_variant & c. $-52+1593 \mathrm{G}>\mathrm{A}$ & \\
\hline 40666198 & $\mathrm{C}$ & $T$ & SNP & intron_variant & c. $-52+1801 C>T$ & \\
\hline 40666293 & C & $\mathrm{T}$ & SNP & intron_variant & c. $-52+1896 C>T$ & \\
\hline 40666446 & G & $\mathrm{C}$ & SNP & intron_variant & c. $-52+2049 \mathrm{G}>\mathrm{C}$ & \\
\hline 40666492 & G & A & SNP & intron_variant & c. $-52+2095 \mathrm{G}>\mathrm{A}$ & \\
\hline 40666509 & G & $\mathrm{C}$ & SNP & intron_variant & c. $-52+2112 \mathrm{G}>\mathrm{C}$ & \\
\hline 40666630 & A & G & SNP & intron_variant & c. $-52+2233 \mathrm{~A}>\mathrm{G}$ & \\
\hline 40667111 & G & A & SNP & intron_variant & c. $-52+2714 \mathrm{G}>\mathrm{A}$ & \\
\hline 40667116 & C & CAGTT & INS & intron_variant & c. $-52+2719-52+2720$ insAGTT & \\
\hline 40667207 & C & $\mathrm{T}$ & SNP & intron_variant & c. $-52+2810 \mathrm{C}>\mathrm{T}$ & \\
\hline 40667521 & $\mathbf{T}$ & $\mathrm{C}$ & SNP & intron_variant & c. $-52+3124 \mathrm{~T}>\mathrm{C}$ & \\
\hline 40667612 & G & $\mathrm{T}$ & SNP & intron_variant & c. $-52+3215 G>T$ & \\
\hline 40667668 & A & G & SNP & intron_variant & c. $-52+3271 \mathrm{~A}>\mathrm{G}$ & \\
\hline 40667702 & $\mathrm{C}$ & $\mathbf{T}$ & SNP & intron_variant & c. $-52+3305 \mathrm{C}>\mathrm{T}$ & \\
\hline 40667801 & A & $\mathrm{T}$ & SNP & intron_variant & c. $-52+3404 \mathrm{~A}>\mathrm{T}$ & \\
\hline 40668796 & $\mathrm{C}$ & G & SNP & intron_variant & c. $-52+4399 \mathrm{C}>\mathrm{G}$ & \\
\hline 40669054 & $\mathbf{A}$ & G & SNP & intron_variant & c. $-52+4657 \mathrm{~A}>\mathrm{G}$ & \\
\hline 40669137 & C & $\mathbf{T}$ & SNP & intron_variant & c. $-52+4740 \mathrm{C}>\mathrm{T}$ & \\
\hline 40669138 & $\mathrm{C}$ & G & SNP & intron_variant & c. $-52+4741 \mathrm{C}>\mathrm{G}$ & \\
\hline 40669255 & $\mathrm{~T}$ & $\mathrm{C}$ & SNP & intron_variant & c. $-52+4858 \mathrm{~T}>\mathrm{C}$ & \\
\hline 40669563 & A & G & SNP & intron_variant & c. $-52+5166 \mathrm{~A}>\mathrm{G}$ & \\
\hline 40669656 & G & A & SNP & intron_variant & c. $-52+5259 \mathrm{G}>\mathrm{A}$ & \\
\hline 40669872 & $\mathrm{C}$ & A & SNP & intron_variant & c. $-52+5475 \mathrm{C}>A$ & \\
\hline 40670027 & $\mathbf{T}$ & C & SNP & intron_variant & c. $-52+5630 \mathrm{~T}>\mathrm{C}$ & \\
\hline 40670115 & A & G & SNP & intron_variant & c. $-52+5718 \mathrm{~A}>\mathrm{G}$ & \\
\hline 40670182 & G & A & SNP & intron_variant & c. $-52+5785 \mathrm{G}>\mathrm{A}$ & \\
\hline 40670557 & A & $\mathrm{C}$ & SNP & intron_variant & c. $-52+6160 \mathrm{~A}>\mathrm{C}$ & \\
\hline 40670609 & $\mathrm{~T}$ & $\mathrm{C}$ & SNP & intron_variant & c. $-52+6212 \mathrm{~T}>\mathrm{C}$ & \\
\hline 40670632 & G & $\mathbf{A}$ & SNP & intron_variant & c. $-52+6235 \mathrm{G}>\mathrm{A}$ & \\
\hline 40670862 & $T$ & G & SNP & intron_variant & c. $-51-6416 \mathrm{~T}>\mathrm{G}$ & \\
\hline 40671036 & $\mathbf{T}$ & C & SNP & intron_variant & c. $-51-6242 \mathrm{~T}>\mathrm{C}$ & \\
\hline 40671340 & A & G & SNP & intron_variant & c. $-51-5938 \mathrm{~A}>\mathrm{G}$ & \\
\hline 40671594 & $\mathrm{C}$ & $\mathrm{T}$ & SNP & intron_variant & c. $-51-5684 C>T$ & \\
\hline 40671648 & $\mathrm{C}$ & $T$ & SNP & intron_variant & c. $-51-5630 \mathrm{C}>\mathrm{T}$ & \\
\hline 40671784 & G & A & SNP & intron_variant & c. $-51-5494 \mathrm{G}>\mathrm{A}$ & \\
\hline 40672081 & G & A & SNP & intron_variant & c. $-51-5197 \mathrm{G}>\mathrm{A}$ & \\
\hline 40672118 & $\mathbf{T}$ & G & SNP & intron_variant & c. $-51-5160 \mathrm{~T}>\mathrm{G}$ & \\
\hline 40672137 & $\mathbf{A}$ & G & SNP & intron_variant & c. $-51-5141 \mathrm{~A}>\mathrm{G}$ & \\
\hline 40672199 & C & $\mathrm{T}$ & SNP & intron_variant & c. $-51-5079 \mathrm{C}>\mathrm{T}$ & \\
\hline 40672314 & G & $\mathbf{A}$ & SNP & intron_variant & c. $-51-4964 \mathrm{G}>\mathrm{A}$ & \\
\hline 40672371 & $\mathrm{AC}$ & $\mathbf{A}$ & DEL & intron_variant & c.-51-4906delC & \\
\hline
\end{tabular}




\begin{tabular}{|c|c|c|c|c|c|c|}
\hline POSITION & REF & ALT & Change_Type & Annotation & HGVS.c & HGVS.p \\
\hline 40672581 & A & C & SNP & intron_variant & c. $-51-4697 \mathrm{~A}>\mathrm{C}$ & \\
\hline 40672611 & A & G & SNP & intron_variant & c. $-51-4667 \mathrm{~A}>\mathrm{G}$ & \\
\hline 40672886 & G & A & SNP & intron_variant & c. $-51-4392 \mathrm{G}>\mathrm{A}$ & \\
\hline 40673144 & CT & C & DEL & intron_variant & c.-51-4133delT & \\
\hline 40673146 & C & A & SNP & intron_variant & c. $-51-4132 \mathrm{C}>\mathrm{A}$ & \\
\hline 40673171 & $\mathrm{C}$ & $\mathbf{T}$ & SNP & intron_variant & c. $-51-4107 \mathrm{C}>\mathrm{T}$ & \\
\hline 40673196 & G & A & SNP & intron_variant & c. $-51-4082 \mathrm{G}>\mathrm{A}$ & \\
\hline 40673207 & C & $\mathbf{T}$ & SNP & intron_variant & c. $-51-4071 \mathrm{C}>\mathrm{T}$ & \\
\hline 40673238 & $\mathbf{T}$ & $\mathrm{C}$ & SNP & intron_variant & c. $-51-4040 \mathrm{~T}>\mathrm{C}$ & \\
\hline 40673601 & G & A & SNP & intron_variant & c. $-51-3677 \mathrm{G}>\mathrm{A}$ & \\
\hline 40673787 & $\mathrm{~T}$ & C & SNP & intron_variant & c. $-51-3491 \mathrm{~T}>\mathrm{C}$ & \\
\hline 40673891 & $T$ & G & SNP & intron_variant & c. $-51-3387 \mathrm{~T}>\mathrm{G}$ & \\
\hline 40673915 & G & A & SNP & intron_variant & c. $-51-3363 \mathrm{G}>\mathrm{A}$ & \\
\hline 40673924 & A & G & SNP & intron_variant & c. $-51-3354 \mathrm{~A}>\mathrm{G}$ & \\
\hline 40673977 & C & $\mathbf{T}$ & SNP & intron_variant & c. $-51-3301 \mathrm{C}>\mathrm{T}$ & \\
\hline 40674013 & ATC & A & DEL & intron_variant & c.-51-3264_-51-3263delTC & \\
\hline 40674022 & CT & $\mathrm{C}$ & DEL & intron_variant & c.-51-3255delT & \\
\hline 40674032 & $T$ & A & SNP & intron_variant & c. $-51-3246 \mathrm{~T}>\mathrm{A}$ & \\
\hline 40674106 & AT & A & DEL & intron_variant & c.-51-3171delT & \\
\hline 40674116 & $T$ & A & SNP & intron_variant & c. $-51-3162 \mathrm{~T}>\mathrm{A}$ & \\
\hline 40674256 & $\mathrm{~T}$ & C & SNP & intron_variant & c. $-51-3022 \mathrm{~T}>\mathrm{C}$ & \\
\hline 40674284 & $\mathrm{C}$ & $\mathbf{T}$ & SNP & intron_variant & c. $-51-2994 \mathrm{C}>\mathrm{T}$ & \\
\hline 40674333 & C & $T$ & SNP & intron_variant & c. $-51-2945 C>T$ & \\
\hline 40674518 & GTTTT & G & DEL & intron_variant & c.-51-2759_51-2756delTTTT & \\
\hline 40674670 & TAAAA & TAA & DEL & intron_variant & c.-51-2605_51-2604delAA & \\
\hline 40674670 & TAAAA & TAAAAAA & INS & intron_variant & c.-51-2604_-51-2603insAA & \\
\hline 40674670 & TAAAA & $\mathrm{T}$ & DEL & intron_variant & c.-51-2607_-51-2604delAAAA & \\
\hline 40674836 & $\mathrm{~T}$ & $\mathrm{C}$ & SNP & intron_variant & c. $-51-2442 \mathrm{~T}>\mathrm{C}$ & \\
\hline 40675117 & C & $\mathbf{T}$ & SNP & intron_variant & c. $-51-2161 \mathrm{C}>\mathrm{T}$ & \\
\hline 40675174 & A & G & SNP & intron_variant & c. $-51-2104 \mathrm{~A}>\mathrm{G}$ & \\
\hline 40675276 & $\mathbf{T}$ & $\mathbf{A}$ & SNP & intron_variant & c. $-51-2002 \mathrm{~T}>\mathrm{A}$ & \\
\hline 40675601 & A & $\mathrm{C}$ & SNP & intron_variant & c. $-51-1677 \mathrm{~A}>\mathrm{C}$ & \\
\hline 40675646 & $\mathbf{A}$ & C & SNP & intron_variant & c. $-51-1632 \mathrm{~A}>\mathrm{C}$ & \\
\hline 40675724 & $\mathrm{~T}$ & G & SNP & intron_variant & c. $-51-1554 \mathrm{~T}>\mathrm{G}$ & \\
\hline 40675826 & C & G & SNP & intron_variant & c. $-51-1452 \mathrm{C}>\mathrm{G}$ & \\
\hline 40676008 & $\mathbf{T}$ & C & SNP & intron_variant & c. $-51-1270 \mathrm{~T}>\mathrm{C}$ & \\
\hline 40676033 & A & G & SNP & intron_variant & c. $-51-1245 \mathrm{~A}>\mathrm{G}$ & \\
\hline 40676267 & A & G & SNP & intron_variant & c. $-51-1011 \mathrm{~A}>\mathrm{G}$ & \\
\hline 40676296 & G & $\mathbf{A}$ & SNP & intron_variant & c. $-51-982 \mathrm{G}>\mathrm{A}$ & \\
\hline 40676298 & A & G & SNP & intron_variant & c. $-51-980 A>G$ & \\
\hline 40676714 & A & G & SNP & intron_variant & c. $-51-564 \mathrm{~A}>\mathrm{G}$ & \\
\hline 40676756 & A & G & SNP & intron_variant & c. $-51-522 \mathrm{~A}>\mathrm{G}$ & \\
\hline 40676882 & C & $\mathbf{T}$ & SNP & intron_variant & c. $-51-396 \mathrm{C}>\mathrm{T}$ & \\
\hline 40676959 & A & $\mathrm{C}$ & SNP & intron_variant & c. $-51-319 \mathrm{~A}>\mathrm{C}$ & \\
\hline 40677282 & G & $T$ & SNP & 5_prime_UTR_variant & c. $-47 \mathbf{G}>\mathbf{T}$ & \\
\hline 40677343 & $\mathrm{C}$ & $\mathbf{T}$ & SNP & synonymous_variant & $c .15 \mathrm{C}>\mathrm{T}$ & p.Ser5Ser \\
\hline 40677451 & $\mathbf{A}$ & G & SNP & synonymous_variant & c. $123 \mathrm{~A}>\mathrm{G}$ & p.Pro41Pro \\
\hline 40677610 & A & G & SNP & intron_variant & c. $228+54 \mathrm{~A}>\mathrm{G}$ & \\
\hline 40677672 & G & $\mathrm{C}$ & SNP & intron_variant & c. $228+116 \mathrm{G}>\mathrm{C}$ & \\
\hline 40677857 & $\mathbf{T}$ & C & SNP & intron_variant & c. $228+301 \mathrm{~T}>\mathrm{C}$ & \\
\hline 40677860 & C & $\mathbf{T}$ & SNP & intron_variant & c. $228+304 \mathrm{C}>\mathrm{T}$ & \\
\hline 40677895 & C & G & SNP & intron_variant & c. $228+339 \mathrm{C}>\mathrm{G}$ & \\
\hline 40677941 & A & G & SNP & intron_variant & c. $228+385 \mathrm{~A}>\mathrm{G}$ & \\
\hline 40677956 & G & $\mathbf{A}$ & SNP & intron_variant & c. $228+400 \mathrm{G}>\mathrm{A}$ & \\
\hline 40677978 & C & G & SNP & intron_variant & c. $228+422 \mathrm{C}>\mathrm{G}$ & \\
\hline 40678016 & C & $\mathbf{A}$ & SNP & intron_variant & c. $228+460 \mathrm{C}>\mathrm{A}$ & \\
\hline 40678046 & C & $\mathbf{T}$ & SNP & intron_variant & c. $228+490 \mathrm{C}>\mathrm{T}$ & \\
\hline 40678145 & $\mathrm{~T}$ & C & SNP & intron_variant & c. $228+589 \mathrm{~T}>\mathrm{C}$ & \\
\hline 40678191 & G & A & SNP & intron_variant & c. $228+635 \mathrm{G}>\mathrm{A}$ & \\
\hline 40678414 & $\mathrm{C}$ & A & SNP & intron_variant & c. $228+858 \mathrm{C}>\mathrm{A}$ & \\
\hline 40678532 & $\mathbf{A}$ & G & SNP & intron_variant & c. $228+976 \mathrm{~A}>\mathrm{G}$ & \\
\hline 40678589 & G & $\mathbf{A}$ & SNP & intron_variant & c. $228+1033 \mathrm{G}>\mathrm{A}$ & \\
\hline 40678771 & G & $\mathbf{A}$ & SNP & intron_variant & c. $228+1215 \mathrm{G}>\mathrm{A}$ & \\
\hline 40678795 & A & G & SNP & intron_variant & c. $228+1239 \mathrm{~A}>\mathrm{G}$ & \\
\hline 40678863 & $T$ & C & SNP & intron_variant & c. $228+1307 \mathrm{~T}>\mathrm{C}$ & \\
\hline 40679202 & C & $\mathbf{T}$ & SNP & intron_variant & c. $228+1646 \mathrm{C}>\mathrm{T}$ & \\
\hline 40679208 & A & G & SNP & intron_variant & $c .228+1652 \mathrm{~A}>\mathrm{G}$ & \\
\hline 40679430 & CCA & $\mathrm{C}$ & DEL & intron_variant & c. $228+1875 \_228+1876 \mathrm{delCA}$ & \\
\hline 40680120 & $\mathrm{~T}$ & G & SNP & intron_variant & c.228+2564T $>\mathrm{G}$ & \\
\hline 40680246 & C & $T$ & SNP & intron_variant & c. $228+2690 \mathrm{C}>\mathrm{T}$ & \\
\hline 40680254 & A & C & SNP & intron_variant & c. $228+2698 \mathrm{~A}>\mathrm{C}$ & \\
\hline 40680381 & $\mathbf{T}$ & G & SNP & intron_variant & c.229-2601T>G & \\
\hline 40680414 & G & A & SNP & intron_variant & c. $229-2568 \mathrm{G}>\mathrm{A}$ & \\
\hline 40680494 & G & $\mathrm{C}$ & SNP & intron_variant & c. $229-2488 \mathrm{G}>\mathrm{C}$ & \\
\hline 40680595 & G & A & SNP & intron_variant & c. $229-2387 \mathrm{G}>\mathrm{A}$ & \\
\hline 40680838 & $\mathbf{A}$ & G & SNP & intron_variant & c. $229-2144 \mathrm{~A}>\mathrm{G}$ & \\
\hline 40681171 & $\mathbf{T}$ & $\mathrm{C}$ & SNP & intron_variant & c.229-1811 $\mathrm{T}>\mathrm{C}$ & \\
\hline 40681213 & $T$ & C & SNP & intron_variant & c.229-1769T $>C$ & \\
\hline 40681305 & G & A & SNP & intron_variant & c.229-1677G>A & \\
\hline 40681350 & A & C & SNP & intron_variant & c.229-1632A $>\mathrm{C}$ & \\
\hline 40681366 & G & $\mathbf{A}$ & SNP & intron_variant & c.229-1616G>A & \\
\hline 40681501 & $T$ & C & SNP & intron_variant & c.229-1481T>C & \\
\hline 40681675 & $T$ & C & SNP & intron_variant & c.229-1307T $>C$ & \\
\hline 40681731 & $\mathrm{~T}$ & $\mathrm{C}$ & SNP & intron_variant & c. $229-1251 \mathrm{~T}>\mathrm{C}$ & \\
\hline
\end{tabular}




\begin{tabular}{|c|c|c|c|c|c|c|}
\hline POSITION & REF & ALT & Change_Type & Annotation & HGVS.c & HGVS.p \\
\hline 40681841 & $\mathbf{A}$ & $\begin{array}{l}\text { AGAGCTCTGTGGACAGAGCTG } \\
\end{array}$ & INS & intron_variant & c.229-1141_229-1140insGAGCTCTGTGGACAGAGCTG & \\
\hline 40681857 & C & T & SNP & intron_variant & c.229-1125C $>\mathrm{T}$ & \\
\hline 40681949 & $\mathrm{~T}$ & C & SNP & intron_variant & c.229-1033T $>C$ & \\
\hline 40682155 & $\mathbf{T}$ & $\mathrm{C}$ & SNP & intron_variant & c.229-827T $>\mathrm{C}$ & \\
\hline 40682225 & $T$ & A & SNP & intron_variant & c.229-757T $>\mathrm{A}$ & \\
\hline 40682262 & A & C & SNP & intron_variant & c. $229-720 \mathrm{~A}>\mathrm{C}$ & \\
\hline 40682282 & $T$ & $\mathrm{C}$ & SNP & intron_variant & c.229-700T $>\mathrm{C}$ & \\
\hline 40682300 & $\mathrm{~T}$ & $\mathrm{C}$ & SNP & intron_variant & c.229-682T>C & \\
\hline 40682423 & ATG & A & DEL & intron_variant & c.229-558_229-557delTG & \\
\hline 40682433 & G & A & SNP & intron_variant & $c .229-549 \mathrm{G}>\mathrm{A}$ & \\
\hline 40682492 & A & G & SNP & intron_variant & c. $229-490 \mathrm{~A}>\mathrm{G}$ & \\
\hline 40682749 & $\mathrm{~T}$ & $\mathrm{C}$ & SNP & intron_variant & c.229-233T $>C$ & \\
\hline 40682798 & $\mathrm{~T}$ & G & SNP & intron_variant & c. $229-184 \mathrm{~T}>\mathrm{G}$ & \\
\hline 40682921 & C & $T$ & SNP & intron_variant & c.229-61C $>\mathrm{T}$ & \\
\hline 40683044 & $\mathbf{T}$ & C & SNP & synonymous_variant & c.291T $>\mathrm{C}$ & p.Ser97Ser \\
\hline 40683308 & G & A & SNP & intron_variant & c. $307-107 \mathrm{G}>\mathrm{A}$ & \\
\hline 40683718 & C & A & SNP & intron_variant & c. $438+45 \mathrm{C}>\mathrm{A}$ & \\
\hline 40684011 & $\mathbf{A}$ & G & SNP & intron_variant & c. $439-63 \mathrm{~A}>\mathrm{G}$ & \\
\hline 40684069 & $T$ & $\mathrm{C}$ & SNP & splice_region_variant & c.439-5T $>C$ & \\
\hline 40684225 & C & G & SNP & intron_variant & c.556+34C>G & \\
\hline 40684324 & C & G & SNP & intron_variant & c.557-70C $>\mathrm{G}$ & \\
\hline 40684516 & C & $\mathbf{T}$ & SNP & intron_variant & c. $655+24 \mathrm{C}>\mathrm{T}$ & \\
\hline 40684538 & $T$ & A & SNP & intron_variant & c. $655+46 \mathrm{~T}>\mathrm{A}$ & \\
\hline 40684570 & C & $\mathbf{T}$ & SNP & intron_variant & c. $655+78 \mathrm{C}>\mathrm{T}$ & \\
\hline 40684599 & C & A & SNP & intron_variant & c. $655+107 \mathrm{C}>\mathrm{A}$ & \\
\hline 40684703 & A & G & SNP & intron_variant & c. $656-18 \mathrm{~A}>\mathrm{G}$ & \\
\hline 40684737 & $\mathrm{~T}$ & $\mathrm{C}$ & SNP & synonymous_variant & c.672T $>\mathrm{C}$ & p.Ser224Ser \\
\hline 40684800 & C & $T$ & SNP & intron_variant & c. $711+24 \mathrm{C}>\mathrm{T}$ & \\
\hline 40684994 & G & A & SNP & intron_variant & c. $712-152 \mathrm{G}>\mathrm{A}$ & \\
\hline 40685003 & A & G & SNP & intron_variant & c. $712-143 \mathrm{~A}>\mathrm{G}$ & \\
\hline 40685006 & C & $\mathbf{T}$ & SNP & intron_variant & c. $712-140 \mathrm{C}>\mathrm{T}$ & \\
\hline 40685016 & $\mathrm{C}$ & $\mathrm{T}$ & SNP & intron_variant & c. $712-130 \mathrm{C}>\mathrm{T}$ & \\
\hline 40685085 & G & A & SNP & intron_variant & c.712-61G $>\mathrm{A}$ & \\
\hline 40685137 & $\mathrm{~T}$ & C & SNP & intron_variant & c.712-9T>C & \\
\hline 40685211 & A & G & SNP & synonymous_variant & n.-1216T $>C$ & p.Glu259Glu \\
\hline 40685273 & $\mathbf{T}$ & C & SNP & intron_variant & c. $804+35 T>C$ & \\
\hline 40685279 & G & A & SNP & intron_variant & c. $804+41 \mathrm{G}>\mathrm{A}$ & \\
\hline 40685334 & C & $\mathbf{T}$ & SNP & intron_variant & c.804+96C $>T$ & \\
\hline 40685366 & C & G & SNP & intron_variant & c. $804+128 \mathrm{C}>\mathrm{G}$ & \\
\hline 40685420 & $\mathrm{~T}$ & C & SNP & intron_variant & c.804+182T $>C$ & \\
\hline 40685433 & GCCC & 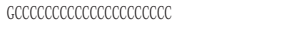 & INS & intron_variant & c.804+198_804+199insCCCCCCCCCCCCCCCCC & \\
\hline 40685433 & GCCC & G & DEL & intron_variant & c.804+196_804+198delCCC & \\
\hline 40685448 & C & G & SNP & intron_variant & c.804+210C $>\mathrm{G}$ & \\
\hline 40685455 & GCC & G & DEL & intron_variant & c.804+218_804+219delCC & \\
\hline 40685667 & $\mathrm{~T}$ & C & SNP & intron_variant & c.805-245T $>C$ & \\
\hline 40686294 & G & A & SNP & intron_variant & c. $963+55 \mathrm{G}>\mathrm{A}$ & \\
\hline 40686386 & A & C & SNP & intron_variant & c. $963+147 \mathrm{~A}>\mathrm{C}$ & \\
\hline 40686425 & $\mathbf{T}$ & C & SNP & intron_variant & c.964-181T>C & \\
\hline 40686449 & $T$ & A & SNP & intron_variant & c.964-157T >A & \\
\hline 40686906 & A & C & SNP & intron_variant & c. $1148+31 \mathrm{~A}>\mathrm{C}$ & \\
\hline 40687035 & G & A & SNP & intron_variant & c. $1148+160 \mathrm{G}>\mathrm{A}$ & \\
\hline 40687082 & G & A & SNP & intron_variant & c. $1148+207 \mathrm{G}>\mathrm{A}$ & \\
\hline 40687135 & G & $\mathbf{A}$ & SNP & intron_variant & c. $1148+260 \mathrm{G}>\mathrm{A}$ & \\
\hline 40687148 & G & GCT & INS & intron_variant & c.1148+273_1148+274insCT & \\
\hline 40687185 & $\mathbf{A}$ & C & SNP & intron_variant & c.1148+310A $>C$ & \\
\hline 40687196 & $\mathrm{~T}$ & A & SNP & intron_variant & c. $1148+321 \mathrm{~T}>\mathrm{A}$ & \\
\hline 40687449 & A & G & SNP & intron_variant & c. $1149-450 \mathrm{~A}>\mathrm{G}$ & \\
\hline 40687659 & $\mathbf{A}$ & G & SNP & intron_variant & c.1149-240A $>\mathrm{G}$ & \\
\hline 40687697 & A & G & SNP & intron_variant & c.1149-202A $>\mathrm{G}$ & \\
\hline 40687705 & G & $\mathbf{A}$ & SNP & intron_variant & c.1149-194G $>A$ & \\
\hline 40687710 & G & A & SNP & intron_variant & c.1149-189G $>\mathrm{A}$ & \\
\hline 40687849 & $\mathbf{T}$ & C & SNP & intron_variant & c.1149-50T $>C$ & \\
\hline 40687888 & $T$ & $\mathrm{C}$ & SNP & intron_variant & c. $1149-11 T>C$ & \\
\hline 40687937 & C & $\mathbf{T}$ & SNP & missense_variant & c. $-3221 \mathrm{C}>\mathrm{T}$ & p.Ser396Leu \\
\hline 40688071 & $T$ & C & SNP & intron_variant & c. $1242+79 \mathrm{~T}>\mathrm{C}$ & \\
\hline 40688129 & G & A & SNP & intron_variant & c. $1242+137 \mathrm{G}>\mathrm{A}$ & \\
\hline 40688216 & $\mathrm{C}$ & A & SNP & intron_variant & c. $1243-183 \mathrm{C}>\mathrm{A}$ & \\
\hline 40688240 & A & $\mathbf{T}$ & SNP & intron_variant & c. $1243-159 \mathrm{~A}>\mathrm{T}$ & \\
\hline 40688250 & C & $T$ & SNP & intron_variant & c. $1243-149 \mathrm{C}>\mathrm{T}$ & \\
\hline 40688258 & G & A & SNP & intron_variant & c. $1243-141 \mathrm{G}>\mathrm{A}$ & \\
\hline 40688570 & $\mathbf{A}$ & $\mathrm{T}$ & SNP & 3_prime_UTR_variant & c. $* 67 \mathrm{~A}>\mathrm{T}$ & \\
\hline 40688645 & $T$ & G & SNP & 3_prime_UTR_variant & c. $* 142 T>G$ & \\
\hline 40688772 & A & G & SNP & 3_prime_UTR_variant & c. $* 269 \mathrm{~A}>\mathrm{G}$ & \\
\hline 40688802 & A & G & SNP & 3_prime_UTR_variant & c. $* 299 \mathrm{~A}>\mathrm{G}$ & \\
\hline 40688826 & G & A & SNP & 3_prime_UTR_variant & c. $* 323 \mathrm{G}>\mathrm{A}$ & \\
\hline 40688920 & $T$ & G & SNP & downstream_gene_variant & c. $* 417 \mathrm{~T}>\mathrm{G}$ & \\
\hline 40689528 & C & $\mathbf{T}$ & SNP & downstream_gene_variant & c. ${ }^{* 1025 C}>\mathrm{T}$ & \\
\hline 40689540 & $\mathrm{C}$ & $\mathbf{T}$ & SNP & downstream_gene_variant & c. $* 1037 \mathrm{C}>\mathrm{T}$ & \\
\hline 40689747 & A & C & SNP & downstream_gene_variant & c. ${ }^{*} 1244 \mathrm{~A}>\mathrm{C}$ & \\
\hline 40689775 & A & G & SNP & downstream_gene_variant & c. ${ }^{*} 1272 \mathrm{~A}>\mathrm{G}$ & \\
\hline 40690016 & $T$ & C & SNP & downstream_gene_variant & c. ${ }^{*} 1513 \mathrm{~T}>\mathrm{C}$ & \\
\hline 40690090 & G & A & SNP & downstream_gene_variant & c. ${ }^{* 1587 G}>A$ & \\
\hline 40690222 & $T$ & C & SNP & downstream_gene_variant & c. ${ }^{*} 1719 \mathrm{~T}>\mathrm{C}$ & \\
\hline 40690464 & $\mathbf{T}$ & G & SNP & downstream_gene_variant & c. ${ }^{*} 1961 \mathrm{~T}>\mathrm{G}$ & \\
\hline
\end{tabular}


Effect of STARD3 polymorphism on beef

\begin{tabular}{|c|c|c|c|c|c|c|}
\hline POSITION & REF & ALT & Change_Type & Annotation & HGVS.c & HGVS.F \\
\hline 40690764 & C & $T$ & SNP & downstream_gene_variant & c. $* 2261 \mathrm{C}>\mathrm{T}$ & \\
\hline 40690991 & G & GC & INS & downstream_gene_variant & c. ${ }^{2488} * 2489$ insC & \\
\hline 40691123 & G & $\mathrm{C}$ & SNP & downstream_gene_variant & c. $* 2620 \mathrm{G}>\mathrm{C}$ & \\
\hline 40691404 & $\mathrm{C}$ & $\mathbf{T}$ & SNP & downstream_gene_variant & c. $* 2901 \mathrm{C}>\mathrm{T}$ & \\
\hline 40691437 & $\mathrm{~T}$ & G & SNP & downstream_gene_variant & c. $* 2934 T>G$ & \\
\hline 40691473 & A & G & SNP & downstream_gene_variant & c. $* 2970 \mathrm{~A}>\mathrm{G}$ & \\
\hline 40691715 & CCAGCTG & C & DEL & downstream_gene_variant & c. $* 3213 \_* 3218$ delCAGCTG & \\
\hline 40692429 & $\mathrm{~T}$ & $\mathrm{C}$ & SNP & downstream_gene_variant & c. $* 3926 \mathrm{~T}>\mathrm{C}$ & \\
\hline 40692592 & G & A & SNP & downstream_gene_variant & c. $* 4089 \mathrm{G}>\mathrm{A}$ & \\
\hline 40692635 & G & A & SNP & downstream_gene_variant & c. ${ }^{*} 4132 \mathrm{G}>\mathrm{A}$ & \\
\hline 40692738 & CTTGG & C & DEL & downstream_gene_variant & c. $* 4236 \_* 4239$ delTTGG & \\
\hline 40692917 & G & $\mathbf{T}$ & SNP & downstream_gene_variant & c. $* 4414 \mathrm{G}>\mathrm{T}$ & \\
\hline 40692971 & $\mathrm{C}$ & $\mathbf{T}$ & SNP & downstream_gene_variant & c. $* 4468 \mathrm{C}>\mathrm{T}$ & \\
\hline 40693046 & G & A & SNP & downstream_gene_variant & c. $* 4543 \mathrm{G}>\mathrm{A}$ & \\
\hline 40693144 & G & A & SNP & downstream_gene_variant & c. $* 4641 \mathrm{G}>\mathrm{A}$ & \\
\hline 40693172 & $\mathrm{~T}$ & $\mathrm{C}$ & SNP & downstream_gene_variant & c. $* 4669 \mathrm{~T}>\mathrm{C}$ & \\
\hline 40693183 & G & A & SNP & downstream_gene_variant & c. $* 4680 \mathrm{G}>\mathrm{A}$ & \\
\hline 40693211 & TCCAGA & $\mathbf{T}$ & DEL & downstream_gene_variant & c. $* 4709+* 4713$ delCCAGA & \\
\hline 40693312 & C & $\mathrm{T}$ & SNP & downstream_gene_variant & c. ${ }^{*} 4809 \mathrm{C}>\mathrm{T}$ & \\
\hline 40693373 & G & A & SNP & downstream_gene_variant & c. $* 4870 G>A$ & \\
\hline 40693422 & G & $\mathbf{T}$ & SNP & downstream_gene_variant & c. $* 4919 G>T$ & \\
\hline 40693569 & CG & $\mathrm{C}$ & DEL & downstream_gene_variant & c. $* 5067 \mathrm{delG}$ & \\
\hline 40693571 & G & $\mathrm{C}$ & SNP & downstream_gene_variant & c. $* 5068 \mathrm{G}>\mathrm{C}$ & \\
\hline 40693607 & G & $\mathbf{T}$ & SNP & downstream_gene_variant & c. $* 5104 \mathrm{G}>\mathrm{T}$ & \\
\hline 40693685 & A & $\mathbf{T}$ & SNP & downstream_gene_variant & c. $* 5182 \mathrm{~A}>\mathrm{T}$ & \\
\hline 40693695 & A & G & SNP & downstream_gene_variant & c. $* 5192 \mathrm{~A}>\mathrm{G}$ & \\
\hline 40693794 & A & G & SNP & downstream_gene_variant & c. $* 5291 \mathrm{~A}>\mathrm{G}$ & \\
\hline 40693879 & C & $\mathrm{T}$ & SNP & downstream_gene_variant & c. $* 5376 \mathrm{C}>\mathrm{T}$ & \\
\hline
\end{tabular}

